Article

\title{
Study on Constitutive Relation of Nickel-Base Superalloy Inconel 718 Based on Long Short Term Memory Recurrent Neural Network
}

\author{
Han Mei ${ }^{1,2}$, Lihui Lang ${ }^{2,3, *}$, Xiaoguang Yang ${ }^{1,2}{ }^{\oplus}$, Zheng Liu $^{3}$ and Xiaoxing $\mathrm{Li}^{3}$ \\ 1 School of Energy and Power Engineering, Beihang University, Beijing 100191, China; \\ mh7196@126.com (H.M.); yxg@buaa.edu.cn (X.Y.) \\ 2 Collaborative Innovation Center of Advanced Aero-Engine, Beijing 100191, China \\ 3 School of Mechanical Engineering and Automation, Beihang University, Beijing 100191, China; \\ zheng.liu.0317@gmail.com (Z.L.); li.xiaoxing@buaa.edu.cn (X.L.) \\ * Correspondence: lang@buaa.edu.cn; Tel.: +86-010-823-16821
}

Received: 14 October 2020; Accepted: 24 November 2020; Published: 27 November 2020

\begin{abstract}
The high temperature tensile test of Inconel 718 under the conditions of deformation temperature of $950{ }^{\circ} \mathrm{C}-1100{ }^{\circ} \mathrm{C}$ and strain rate of $0.0005 \mathrm{~s}^{-1}-0.1 \mathrm{~s}^{-1}$ was carried out, and its true stress-true strain curve was drawn. Through the analysis of the flow stress of Inconel 718 under different conditions, it can be seen that the high-temperature rheological behavior of Inconel 718 is affected by the coupling of strain hardening effect and dynamic softening effect, and has significant loading history correlation. By applying the stretched data, a long short term memory (LSTM) recurrent neural network was trained to characterize the constitutive relationship of Inconel 718. The experimental results show that the prediction results of the LSTM constitutive model are extremely consistent with the experimental data, which is significantly better than the modified Johnson-Cook (M-JC) model. Finally, high temperature tensile experiments under variable strain rates were carried out to verify the feasibility of the LSTM constitutive model in the complex loading and unloading stages.
\end{abstract}

Keywords: Inconel 718; constitutive model; long short term memory (LSTM); high-temperature stretching; recurrent neural network

\section{Introduction}

Inconel 718 is currently the most widely used nickel-based superalloy, it has good comprehensive mechanical properties and mechanical stability below $650^{\circ} \mathrm{C}$ [1,2]. The main strengthening mechanism of Inconel 718 is precipitation strengthening. The main strengthening phase is $\gamma^{\prime \prime}(\mathrm{Ni3Nb})$, supplemented by the weak strengthening phase $\gamma$ (Ni3AlTi) with a face-centered cubic structure [3]. The $\gamma^{\prime \prime}$ phase is metastable, and its equilibrium phase is the $\delta$ phase $(\mathrm{Ni3Nb})$ with orthogonal structure [4]. Compared with other types of precipitation hardening alloys, Inconel 718 has a larger coherent distortion between the precipitation strengthening phase $\gamma^{\prime \prime}$ phase and the matrix, which makes the structure and properties of the alloy more stable [5,6]. Due to its good high-temperature strength, oxidation resistance, thermal corrosion resistance and fatigue performance, Inconel 718 is widely used in the manufacture of hot parts of aero engines and high temperature parts of aerospace rocket engines [7]. Meanwhile, Inconel 718 is also recognized as a difficult-to-form material. It has extremely high resistance to high temperature deformation, and its alloy structure is particularly sensitive to the forming process. In most hot forming and welding processes, Inconel 718 has the problems of high process temperature requirement and narrow selection of process parameters [8]. At present, in order to save material and experimental costs, finite element analysis of the forming process has become the main method to evaluate the formability 
of Inconel 718. The reliability of the finite element method mainly depends on the constitutive model that can characterize the dynamic rheological properties of the material [9].

In the deformation process of Inconel 718, there are two competing mechanisms: The work hardening and the softening induced by dynamic recovery and dynamic recovery stabilization [10]. These two mechanisms are related to strain, strain rate and temperature, and the constitutive model can characterize the correlation. In review of high temperature constitutive models for metals and alloys, Lin divided the current constitutive models into three categories: Phenomenological constitutive model, physical based constitutive model and artificial neural network (ANN) constitutive model [11]. The basis of the phenomenological constitutive model is the experimental observation of the flow stress under different loading conditions, it does not have strict physical meaning. This type of model is widely used in finite element analysis (FEA) of metal forming process because it contains fewer material parameters and is easy to measure [12]. In order to facilitate the solution of material constants, early studies on the phenomenological constitutive model of Inconel 718 assumed that the effects of strain, strain rate and temperature on rheological behavior are independent, which significantly reduces the prediction accuracy of the model [13-15]. In order to study the coupling effect of strain rate and temperature, Wang et al. modified the Johnson-Cook model and determined the strain rate influencing factors under different rates and temperature conditions through nonlinear regression methods [16]. Mirza Z.H et al. equated the flow stress of Inconel 718 to the effects of strain, strain rate, temperature and their interactions, and established six influence functions as $f_{\varepsilon}, f_{\varepsilon-\dot{\varepsilon}}, f_{\dot{\varepsilon}}, f_{\dot{\varepsilon}-T}, f_{T}$ and $f_{\varepsilon-T}$. The weight of each function is solved by double multivariate nonlinear regression [17]. Lin et al. established a modified Arrhenius model of Inconel 718 taking the effects of multi-factor coupling into consideration. The material constants in this model are the function of the Zener-Hollomon parameter. These studies showed that the coupling effect between strain, strain rate, temperature and other parameters is nonlinear, and it is inaccurate to use traditional regression methods to obtain material constants. Therefore, many researchers used neural network methods to analyze the rheological properties of materials to overcome the limitations of traditional regression methods in solving highly nonlinear problems. Huang and Blackwell measured the flow stress data of Inconel 718 at a temperature range of $950{ }^{\circ} \mathrm{C}$ to $980^{\circ} \mathrm{C}$ and strain rates of $10^{-4} \mathrm{~s}^{-1}$ and $10^{-3} \mathrm{~s}^{-1}$, and used the data to train an ANN constitutive model. Although this model is limited by a small sample size, it has potential in characterizing material work hardening and dynamic softening phenomena [18]. Yao et al. conducted a single impact compression test of Inconel 718 at a temperature of $950-1050{ }^{\circ} \mathrm{C}$ and a strain rate of $0.004-10 \mathrm{~s}^{-1}$. The prediction ability of the single hidden layer BP (Back propagation) neural network model based on the experimental results is significantly better than the modified Arrhenius constitutive model [19]. Bhekisipho Twala introduced the aging time of Inconel 718 into the input variable to characterize the microstructure of the material. The ANN constitutive model trained by this four-dimensional input data set can accurately predict the flow stress of Inconel 718 in multiple initial microstructure states [20].

With the promotion of the phenomenological constitutive model in FEA of various processes, some complex porous lattice structure parts in the aerospace field have also begun to be manufactured using Inconel 718, such as honeycomb structure, lattice truss structure and corrugated board structure [21,22]. In order to meet the lightweight standards in the aerospace field and some special heat dissipation requirements, these structures are usually designed with complex flow channels and micro-support structures, and these structures may become unstable if they undergo slight thermal deformation. In order to weigh the forming quality and the degree of deformation in the hot forming process, modern hot forming processes use complex loading paths to achieve multiple process target control. For example, the loading process of the diffusion bonding process of Inconel 718 includes multiple stages such as plastic deformation, creep and stress relaxation. As mentioned above, the current phenomenological constitutive model and ANN constitutive model of Inconel 718 are mostly based on the total strain theory of plasticity. They mainly focus on the flow stress $\sigma$ under 
the coupling action of strain $\varepsilon$, temperature $T$ and strain rate $\dot{\varepsilon}$. The corresponding relationship can be expressed as:

$$
\sigma=f(\varepsilon, \dot{\varepsilon}, T)
$$

Obviously, these models are only suitable for simple loading situations where the strain component changes proportionally, ignoring the deformation history effect. However, Inconel 718 is a typical viscoplastic material at high temperatures, and its rheological properties are obviously affected by the loading history. Under the complex loading path, the temperature $T$ and strain rate $\dot{\varepsilon}$ should be expressed as time-dependent dynamic functions $T(t)$ and $\dot{\varepsilon}(t)$, then the flow stress at any time $t_{0}$ can be expressed as follows:

$$
\sigma=f\left(\varepsilon\left(t_{0}\right), \dot{\varepsilon}\left(t_{0}\right), T\left(t_{0}\right), \Psi\right)
$$

$\Psi$ is an internal variable function which characterizes the microstructure change of material from the beginning of loading to the time $t_{0}$, which is related to the integral of strain, strain rate and temperature along the loading curve. If the time increment on the loading path is assumed to be $\Delta t$, then at a certain time $t_{0}$ the flow stress is not only related to the loading conditions at that time, but also to the loading conditions of $\left(t_{0}-\Delta t\right)$. It is difficult for ANN model to obtain satisfactory results for the prediction of time-dependent sequence data. This is because there is no correlation between the inputs of ANN model, and its outputs are independent of each other. The recurrent neural network (RNN) model is a kind of neural network which takes sequence data as input and recurses in the evolution direction of sequence. Different from ANN model, the hidden layer nodes of the model are connected, and the input of the hidden layer is the superposition of the output of the current input layer and the output of the previous hidden layer [23]. In other words, when RNN model calculates the output of a certain time on the time series, it not only considers the input of the time point, but also considers the memory of the output of the previous time point. Obviously, many RNN models have advantages in learning nonlinear features of sequences. Among them, the long short term memory (LSTM) recurrent neural network, as a specially designed RNN model, effectively avoids the long-term dependency problem of RNN model, so it is widely used in the prediction of various time series [24].

In this study, several groups of Inconel 718 were created and their uniaxial tensile tests at different temperatures and strain rates were carried out, and a LSTM constitutive model was trained based on the experimental results. Compared with the prediction results of modified Johnson-Cook (M-JC) model obtained by experimental parameters fitting, the root mean square error (RMSE) of rheological properties prediction results of the two models under different loading conditions were obtained. In addition, based on the hot forming process curve of Inconel 718 in project, a high temperature tensile test under a complex loading path was designed. The obtained experimental data is compared with the prediction results of LSTM constitutive model under the same path, and the possibility of LSTM constitutive model applied to the flow stress prediction of Inconel 718 under complex loading path was studied.

\section{Experiments}

Commercial hot-rolled Inconel 718 sheet material with a thickness of $3 \mathrm{~mm}$ was selected as the blank of the high temperature tensile test specimen, which was purchased from Sunrise Metal Technology (Beijing, China) Co. Ltd. The material element composition and ratio are shown in Table 1. Wire cut electrical discharge machining (EDM) equipment was used to prepare tensile test pieces, the dimensions of these specimens are shown in Figure 1, where the axial direction is the rolling direction of the sheet. To prevent Inconel 718 from being oxidized at high temperature, zirconia powder was applied to the sample surface. 
Table 1. Chemical composition (wt.\%) of Inconel 718.

\begin{tabular}{cccccccccccc}
\hline $\mathbf{C}$ & $\mathbf{S i}$ & $\mathbf{C r}$ & $\mathbf{N i}$ & $\mathbf{M o}$ & $\mathbf{N b}$ & $\mathbf{T i}$ & $\mathbf{A l}$ & $\mathbf{F e}$ & $\mathbf{B}$ & $\mathbf{Z r}$ & $\mathbf{N}$ \\
\hline 0.002 & 0.31 & 18.25 & 53.6 & 3.1 & 4.12 & 0.96 & 0.6 & 18.34 & 0 & 0 & 0.718 \\
\hline
\end{tabular}

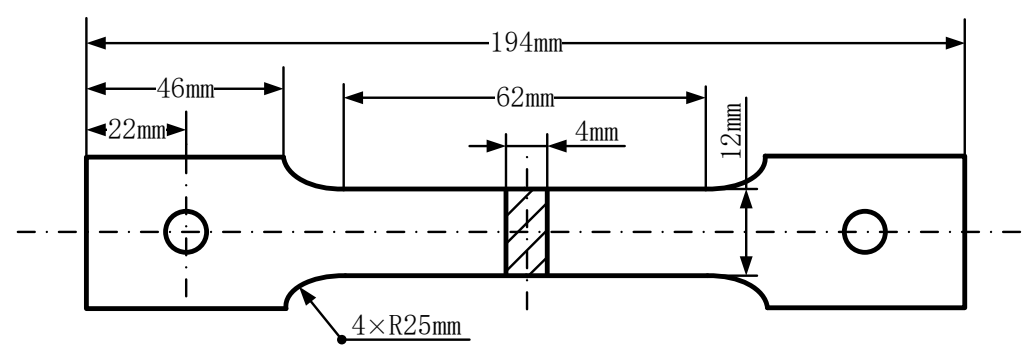

Figure 1. Schematic diagram of specimen size for tensile test (unit: $\mathrm{mm}$ ).

The high temperature tensile testing machine (Strong Association Instrument Technology Co. Ltd, Shanghai, China) was used for the test, and the maximum strain obtained was 0.6. The experimental temperature was set at $900^{\circ} \mathrm{C}, 950^{\circ} \mathrm{C}, 1000^{\circ} \mathrm{C}, 1050^{\circ} \mathrm{C}$ and $1100^{\circ} \mathrm{C}$, and the strain rate of $0.1 \mathrm{~s}^{-1}, 0.01 \mathrm{~s}^{-1}$, $0.001 \mathrm{~s}^{-1}$ and $0.0005 \mathrm{~s}^{-1}$. The experimental equipment adopts three-stage temperature measurement technology, which can effectively ensure that the parts are heated evenly. The temperature was increased at a rate of $20^{\circ} \mathrm{C} / \mathrm{min}$ firstly, and the temperature was kept for $10 \mathrm{~min}$ after the three temperature sensors reached the set temperature, and then the specimen was loaded at a constant strain rate. After the experiment, it was taken out when it cooled to room temperature.

\section{Result and Discussion}

\subsection{Flow Stress Curve Analysis of Inconel 718}

Through the high temperature tensile experiments of Inconel 718, engineering stress-engineering strain curves under different temperature and strain rate conditions were obtained. For the convenience of research, equations (3) and (4) are usually used to convert the engineering stress $\sigma_{e}$ and engineering strain $\varepsilon_{e}$ of the material into true stress $\sigma$ and true strain $\varepsilon$, respectively.

$$
\begin{gathered}
\sigma=\sigma_{\mathcal{e}}\left(1+\varepsilon_{e}\right) \\
\varepsilon=\ln \left(1+\varepsilon_{e}\right)
\end{gathered}
$$

Many studies have pointed out that this method has low accuracy in predicting the rheological behavior of materials after necking. According to previous research results [25], an FE-based inverse modeling procedure was used in this paper to derive the true stress-strain curve of Inconel 718 as shown in Figure 2.

It can be seen from the Figure 2 that the stress-strain curves of Inconel 718 at various temperatures have a close changing trend. In the initial stage of deformation, the flow stress increases significantly with the increase of strain, and the work hardening effect caused by the increase of dislocation density is significant. As the deformation continues, the dynamic softening effect inside the material gradually increases, and the dislocation density begins to show a downward trend. The two effects reach equilibrium at a certain deformation moment, at which time the flow stress will tend to stabilize. Obviously, the level of flow stress is affected by the coupling of temperature and strain rate. Under the same temperature conditions, the deformation resistance of Inconel 718 decreases significantly as the strain rate decreases. This is because the deformation time is shortened and the nucleation number of dynamic recrystallization is reduced under high strain rate, which leads to insufficient dynamic softening process of the material. On the other hand, the increase of temperature promotes the slip and climb of dislocations, thereby it improves the nucleation rate of dynamic 
recrystallization and promotes the dynamic softening process of the material. This also explains the phenomenon that the flow stress increases significantly with the decrease of temperature under the same strain rate.

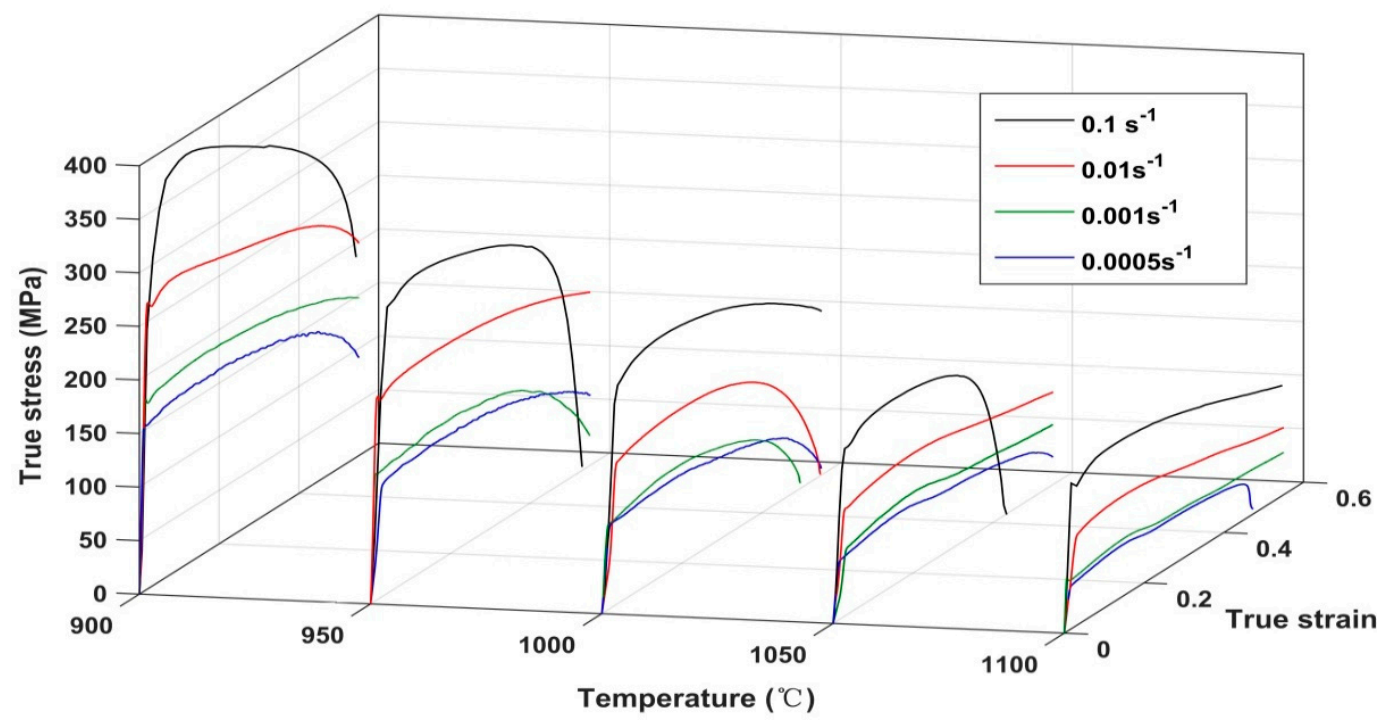

Figure 2. The true stress-strain curve of Inconel 718 at different temperatures and strain rates.

Obviously, due to the complex superposition state of the material's internal hardening effect and dynamic softening effect in the time domain, Inconel 718 exhibits different deformation resistance levels under different deformation conditions, which can be expressed as a set of independent continuous curves. When the actual loading curve changes at a certain moment, the flow stress not only steps on different continuous curves, but also the influence of the superposition state of the material at that moment should be considered. Therefore, it is necessary to establish a constitutive model considering the loading history effect based on the tensile experimental data under different deformation conditions to predict the flow stress at any time in the complex loading curve.

\subsection{The Structure of LSTM Constitutive Model}

The cyclic neural network is a fully connected neural network with a feedback mechanism. There are node connections not only between layers, but also across the timeline. Its structure is shown in Figure 3.

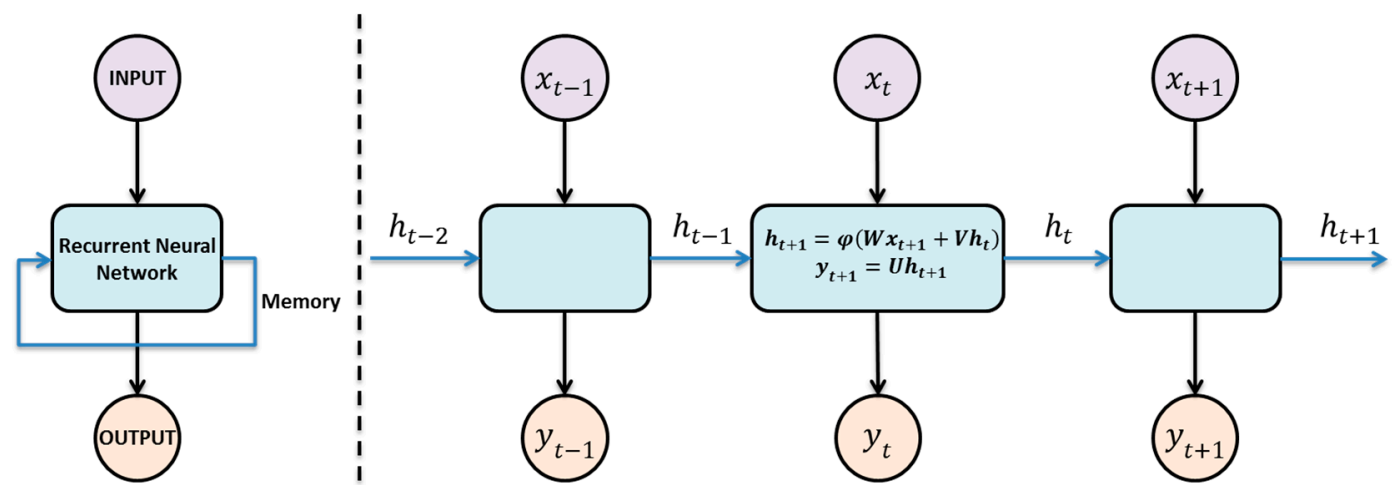

Figure 3. The structure of recurrent neural network (RNN) model. 
The value of the hidden layer neuron of the RNN at a certain moment will be stored inside the neuron, which is called memory $h_{t}$. When calculating the output $y_{t+1}$ of the neuron at the next moment, the memory will work together with the input $x_{t+1}$ at that moment. It can be expressed as:

$$
\begin{gathered}
h_{t+1}=\varphi\left(W x_{t+1}+V h_{t}\right) \\
y_{t+1}=U h_{t+1}
\end{gathered}
$$

in the equation, $W$ is the input transfer function, $V$ is the memory transfer function, $U$ is the output transfer function and $\varphi$ is the activation function. The memory is updated with iteration, but it is assigned different weights. Since the output of the neuron is obtained through the activation of function, whenever the memory is iterated with other inputs, part of the information it contains will be lost. In fact, after four to five iterations, all the memory information will be lost. This is the long-term dependencies problem of the RNN model [24].

For the complex loading curve of Inconel 718, a sudden change and recovery of conditions such as temperature and strain rate occur at a certain moment. When the RNN model is used to forecast the flow stress and if the prediction time is far from the sudden change time, the memory information of the sudden change is lost. The historical effect of this mutation is ignored.

The LSTM network is a cyclic neural network with a special structure design, which is specialized in the ability to remember long-term information, so as to avoid the loss of information in the propagation process. The LSTM network is also composed of multiple groups of repeated neuron modules, and its internal structure is shown in Figure 4.

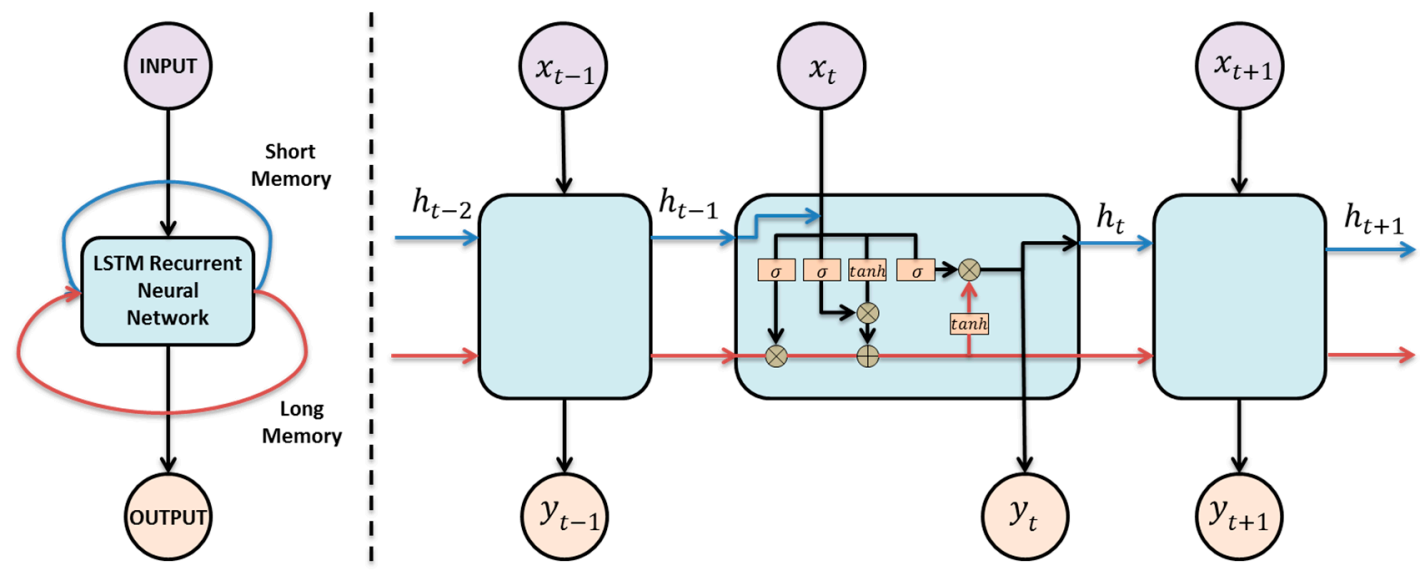

Figure 4. The structure of long short term memory (LSTM) model.

The most notable feature of the LSTM network is that there is a data route that runs through all neuron modules, which is used to transfer the memory state. The memory route is transmitted in parallel with the input-output route of the neuron, and they interact linearly through a specially designed "gate" structure to ensure the stability of the memory information state. Through the "gate" structure, the memory information can be partially deleted or updated, and stored as long-term memory (cell state) and short-term memory (hidden state) by LSTM network. Taking the LSTM network model structure in Figure 4 as an example, when the input value $x_{t}$ at a certain moment and the memory $h_{t-1}$ at the previous moment enter the neuron structure, the forgetting vector $f_{t}$ that determines the degree of memory retention and is generated through the memory gate is:

$$
f_{t}=\sigma\left(W_{f} \times\left[x_{t}, h_{t-1}\right]+b_{f}\right)
$$


Selecting and uploading those memory states that need to be updated in input gate:

$$
\begin{gathered}
i_{t}=\sigma\left(W_{i} \times\left[x_{t}, h_{t-1}\right]+b_{i}\right) \\
\widetilde{C}_{t}=\tanh \left(W_{C} \times\left[x_{t}, h_{t-1}\right]+b_{C}\right)
\end{gathered}
$$

in the equation, $i_{t}$ is the saved vector that determines the update state and $\widetilde{C}_{t}$ is the value required to be updated. Through simple linear calculation of memory gate and input gate, the updated memory state $C_{t}$ can be obtained:

$$
C_{t}=f_{t} \times C_{t-1}+i_{t} \times \widetilde{C}_{t}
$$

Finally, the output of the neuron structure comes from the linear transformation of $C_{t}$ and $\left[x_{t}, h_{t-1}\right]$ through the output gate:

$$
\begin{gathered}
O_{t}=\sigma\left(W_{O} \times\left[x_{t}, h_{t-1}\right]+b_{O}\right) \\
h_{t}=O_{t} \times \tanh \left(C_{t}\right)
\end{gathered}
$$

According to the forward propagation principle of the aforementioned LSTM network, this article built a LSTM network model for predicting the constitutive relationship of Inconel 718. The model consists of an input layer containing three nodes, a LSTM hidden layer containing 100 nodes, two fully connected layers containing 50 nodes, a dropout layer and a regression layer.

\subsection{Training of the LSTM Constitutive Model}

Then, 20 sets of true stress-true strain data obtained by Inconel 718 uniaxial tensile experiment were used for training of LSTM network. Two sets of data under the conditions of $1000^{\circ} \mathrm{C}, 0.1 \mathrm{~s}^{-1}$ and $1100{ }^{\circ} \mathrm{C}, 0.0005 \mathrm{~s}^{-1}$ were selected as the validation set, and the remaining data were used as the training set. In the tensile experiments, in order to ensure the same time interval between each data point, the experimental equipment used a fixed acquisition frequency, which lead to a large difference in the length of the experimental data under different strain rates. The data length under the strain rate of $0.001 \mathrm{~s}^{-1}$ at the same temperature is 100 times that of $0.1 \mathrm{~s}^{-1}$. To facilitate data processing, the experimental data was truncated with 700 as the length threshold, and 18 groups of experimental data were divided into 72 training set samples.

In order to alleviate the over-fitting problem of the LSTM recurrent neural network, the dropout method was used to randomly invalidate $50 \%$ of the hidden nodes in each iteration. The invalidated neuron nodes no longer participated in the forward calculation in this iteration and the weight and bias terms were not updated.

The Adam optimization algorithm, that can update the weight of LSTM iteratively based on the training set, and design independent adaptive learning rate for different parameters by calculating the first and second moment estimation of gradient, was adopted as the network training algorithm. The training initial learning rate was set as 0.01 , and the maximum number of iterations was 1000 times. Through 603 iterations, the RMSE of LSTM network reached 2.429.

\subsection{Prediction Results of LSTM Constitutive Model}

The rheological properties of Inconel 718 at different temperatures and strain rates were predicted using the LSTM constitutive model after training. The results are shown in Figure 5.

It can be seen that the fitting results of LSTM constitutive model are in good agreement with the measured results. The correlation coefficient $(R)$ of the fitting results and measurement results under different deformation conditions was calculated by formula (13) and listed in Table 2.

$$
R=\frac{\sum_{i=1}^{N}\left(\sigma_{i}-\bar{\sigma}\right)\left(\delta_{i}-\bar{\delta}\right)}{\sqrt{\sum_{i=1}^{N}\left(\sigma_{i}-\bar{\sigma}\right)^{2} \sum_{i=1}^{N}\left(\delta_{i}-\bar{\delta}\right)^{2}}}
$$



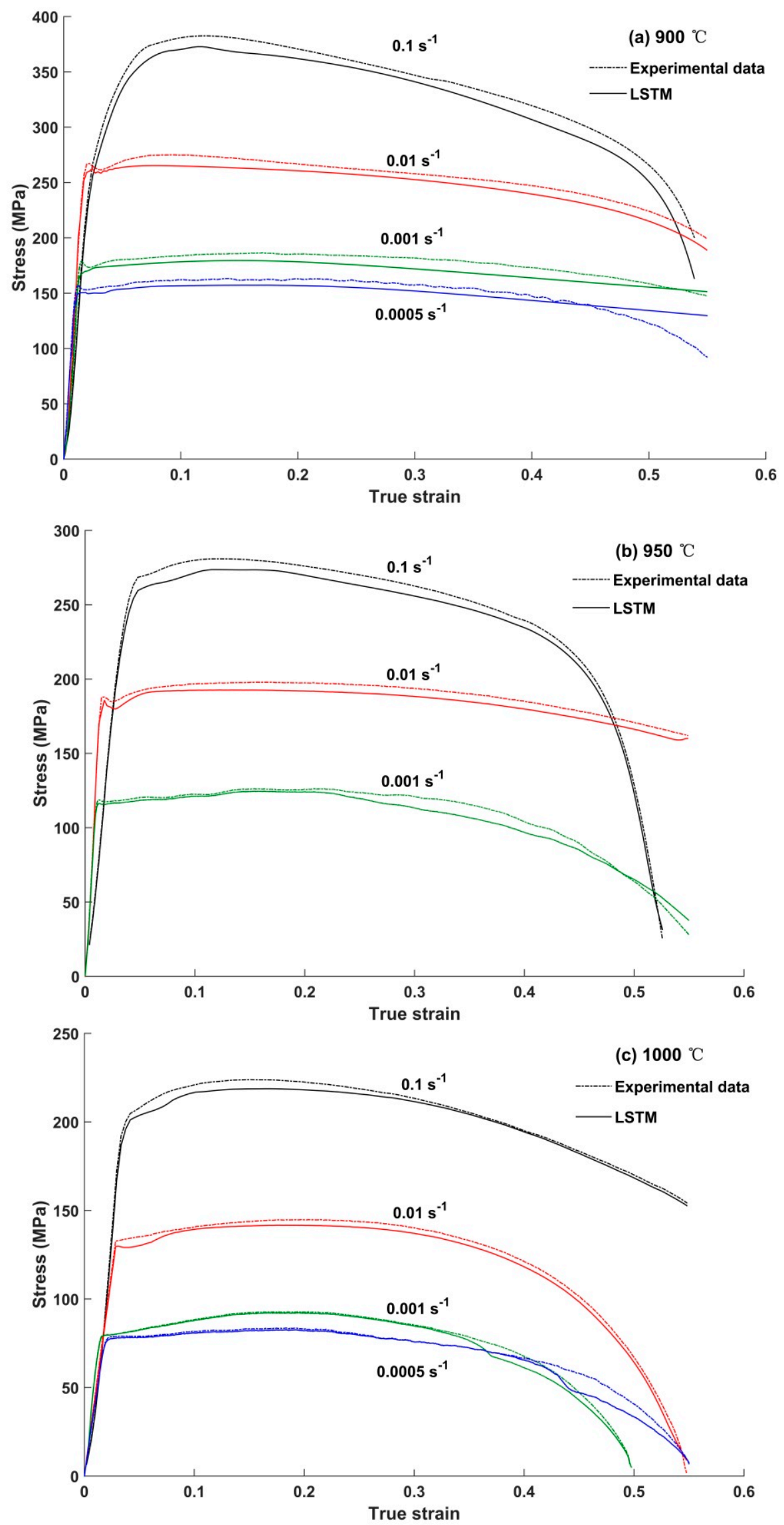

Figure 5. Cont. 

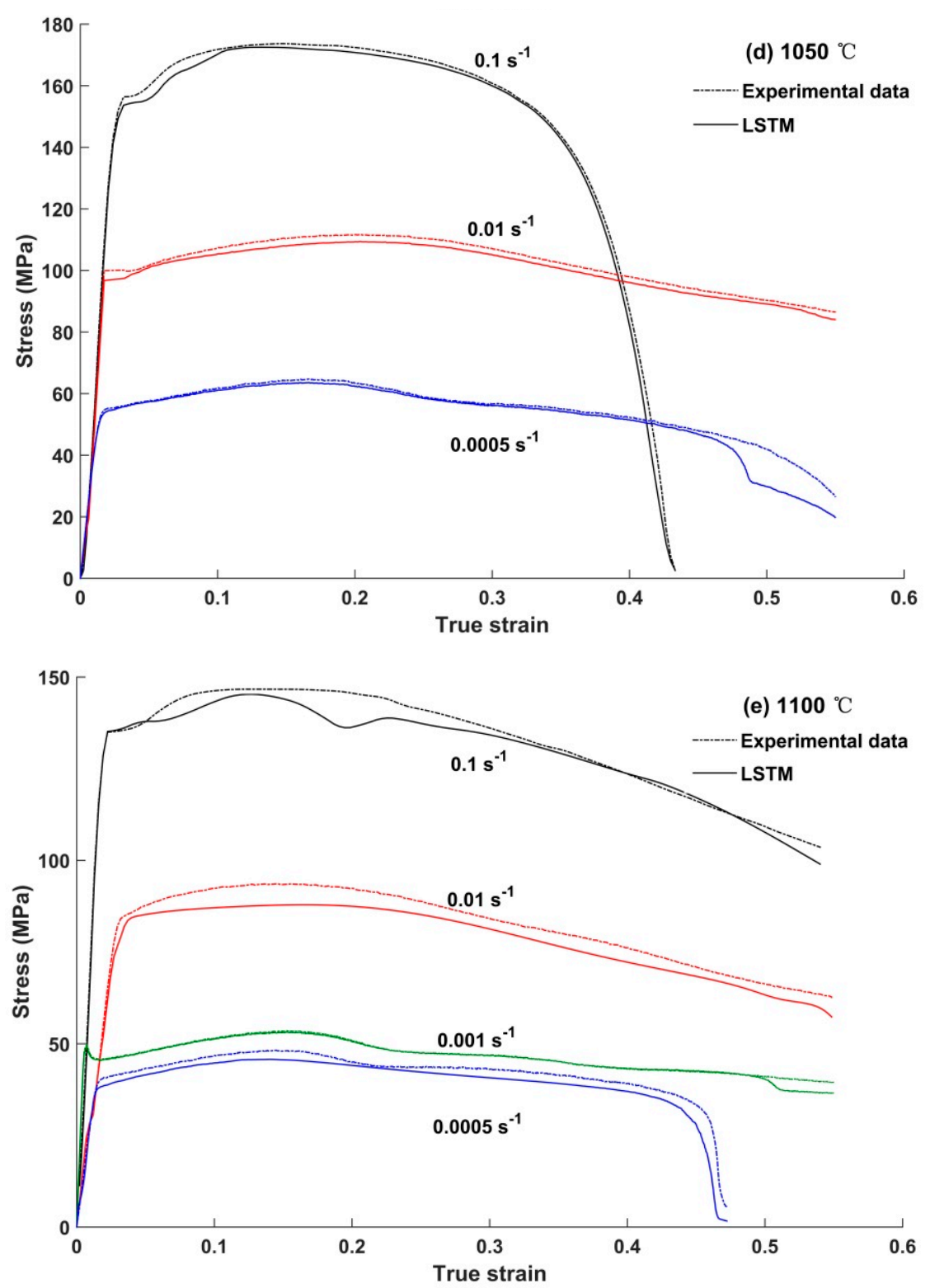

Figure 5. Fitting results of LSTM constitutive model. (a) $900{ }^{\circ} \mathrm{C}$; (b) $950{ }^{\circ} \mathrm{C}$; (c) $1000{ }^{\circ} \mathrm{C}$; (d) $1000{ }^{\circ} \mathrm{C}$; (e) $1100{ }^{\circ} \mathrm{C}$.

Table 2. The correlation coefficients between measured and predicted values.

\begin{tabular}{cccccc}
\hline Deformation Parameters & $\mathbf{9 0 0}{ }^{\circ} \mathbf{C}$ & $\mathbf{9 5 0}{ }^{\circ} \mathbf{C}$ & $\mathbf{1 0 0 0}{ }^{\circ} \mathbf{C}$ & $\mathbf{1 0 5 0}{ }^{\circ} \mathbf{C}$ & $\mathbf{1 1 0 0}{ }^{\circ} \mathbf{C}$ \\
\hline $0.1 \mathrm{~s}^{-1}$ & 0.99972 & 0.99990 & 0.99986 & 0.99991 & 0.99980 \\
$0.01 \mathrm{~s}^{-1}$ & 0.99987 & 0.99990 & 0.99996 & 0.99993 & 0.99989 \\
$0.001 \mathrm{~s}^{-1}$ & 0.99915 & 0.99703 & 0.99459 & - & 0.99507 \\
$0.0005 \mathrm{~s}^{-1}$ & 0.99691 & - & 0.99147 & 0.99083 & 0.99933 \\
\hline
\end{tabular}

In order to further verify the prediction accuracy of LSTM model, the prediction results at $1000{ }^{\circ} \mathrm{C}$, $0.1 \mathrm{~s}^{-1}$ and $1100{ }^{\circ} \mathrm{C}, 0.0005 \mathrm{~s}^{-1}$ were compared with the results of M-JC model. The Johnson-Cook model is a typical phenomenological constitutive model, which can effectively characterize the independent effects of temperature, strain rate and strain on flow stress. Taking the coupling effect between 
deformation conditions into consideration, the modified Johnson-Cook model [26,27] was proposed. It can be expressed as:

$$
\sigma=\left(A_{1}+B_{1} \varepsilon+B_{2} \varepsilon^{2}\right)\left(1+C_{1} \ln \frac{\dot{\varepsilon}}{\dot{\varepsilon}_{r e f}}\right) \times \exp \left[\left(\lambda_{1}+\lambda_{2} \ln \frac{\dot{\varepsilon}}{\dot{\varepsilon}_{r e f}}\right)\left(T-T_{r e f}\right)\right]
$$

where $\sigma$ is the flow stress of the material, $\varepsilon$ is the strain, and $\dot{\varepsilon}$ and $\dot{\varepsilon}_{r e f}$ is strain rate and reference strain rate respectively, $T$ and $T_{\text {ref }}$ is temperature and reference temperature respectively, $A_{1}, B_{1}, B_{2}, C_{1}, \lambda_{1}$ and $\lambda_{2}$ are material constants. Based on the measured data of Inconel 718 high temperature tensile tests, the unknown material constants of M-JC model can be obtained by linear fitting method. Taking $900{ }^{\circ} \mathrm{C}$ as the reference temperature and $0.001 \mathrm{~s}^{-1}$ as the reference strain rate, the M-JC model using the procedure modified by Lin et al. [26] of Inconel 718 can be deduced:

$$
\begin{aligned}
\sigma=\left(148.2+370.6 \varepsilon-896.6 \varepsilon^{2}\right)\left(1+0.1985 \times \ln \frac{\dot{\varepsilon}}{0.001}\right) \\
\quad \times \exp \left[\left(-0.007177+0.000402 \times \ln \frac{\dot{\varepsilon}}{0.001}\right)(T-900)\right]
\end{aligned}
$$

Figure 6 shows the prediction curves of LSTM constitutive model and M-JC model under different temperature and strain rates. It can be seen that the LSTM constitutive model has high prediction accuracy in the elastic stage of high temperature deformation. Its RMSE is 0.034 at $950{ }^{\circ} \mathrm{C}, 0.0005 \mathrm{~s}^{-1}$ and 0.028 at $1050{ }^{\circ} \mathrm{C}, 0.001 \mathrm{~s}^{-1}$. In the plastic deformation stage, especially before the necking of specimen, LSTM constitutive model has significant advantages in predicting the flow stress. Table 3 shows the root mean square error and the correlation coefficient of the plasticity stage predicting results

\begin{tabular}{|c|c|c|c|c|}
\hline \multirow{2}{*}{ Performance } & \multicolumn{2}{|c|}{ LSTM Constitutive Model } & \multicolumn{2}{|c|}{ M-JC Constitutive Model } \\
\hline & $950^{\circ} \mathrm{C}, 0.0005 \mathrm{~s}^{-1}$ & $1050{ }^{\circ} \mathrm{C}, 0.001 \mathrm{~s}^{-1}$ & $950{ }^{\circ} \mathrm{C}, 0.0005 \mathrm{~s}^{-1}$ & $1050{ }^{\circ} \mathrm{C}, 0.001 \mathrm{~s}^{-1}$ \\
\hline RMSE & 3.608 & 1.552 & 11.183 & 7.816 \\
\hline $\mathrm{R}$ & 0.99623 & 0.98886 & 0.90218 & 0.81234 \\
\hline
\end{tabular}
of two constitutive models at $950{ }^{\circ} \mathrm{C}, 0.0005 \mathrm{~s}^{-1}$ and $1050{ }^{\circ} \mathrm{C}, 0.001 \mathrm{~s}^{-1}$.

Table 3. Comparison of prediction performance between LSTM and M-JC in plasticity stage.

\subsection{Validation of Prediction Performance of LSTM Constitutive Model under Complex Loading Paths}

In order to verify the ability of the trained LSTM constitutive model to predict the flow stress changes under complex loading paths, the high temperature tensile experiment including loading and unloading stages under different strain rates were designed. The process parameter curve is shown in Figure 7a. The drawing experiment fully refers to the hot forming process curve of Inconel 718 in the actual project, and the comparison between the measured result and the predicted curve of LSTM rigid model is shown in Figure 8.

It can be seen that compared to the M-JC model and other phenomenological constitutive models, the LSTM constitutive model still has high prediction accuracy when the load, unloading and strain rates change suddenly. The RMSE of the prediction results are 2.763. In addition, the LSTM constitutive model has significant advantages in predicting the elastic deformation stage of Inconel 718, the RMSE is 0.075 and 0.064 . It can also be found from the results that the prediction model has large errors in the prediction of Inconel 718's creep phase and stress relaxation phase. This is because the training data was obtained under the condition of constant rate stretching, and there is less data in the viscoelastic phase. By improving the cell structure of the LSTM model and incorporating the standard creep test data into the training set, the accuracy of the model's prediction of the Inconel 718 viscoelastic phase rheological properties can be significantly improved. 

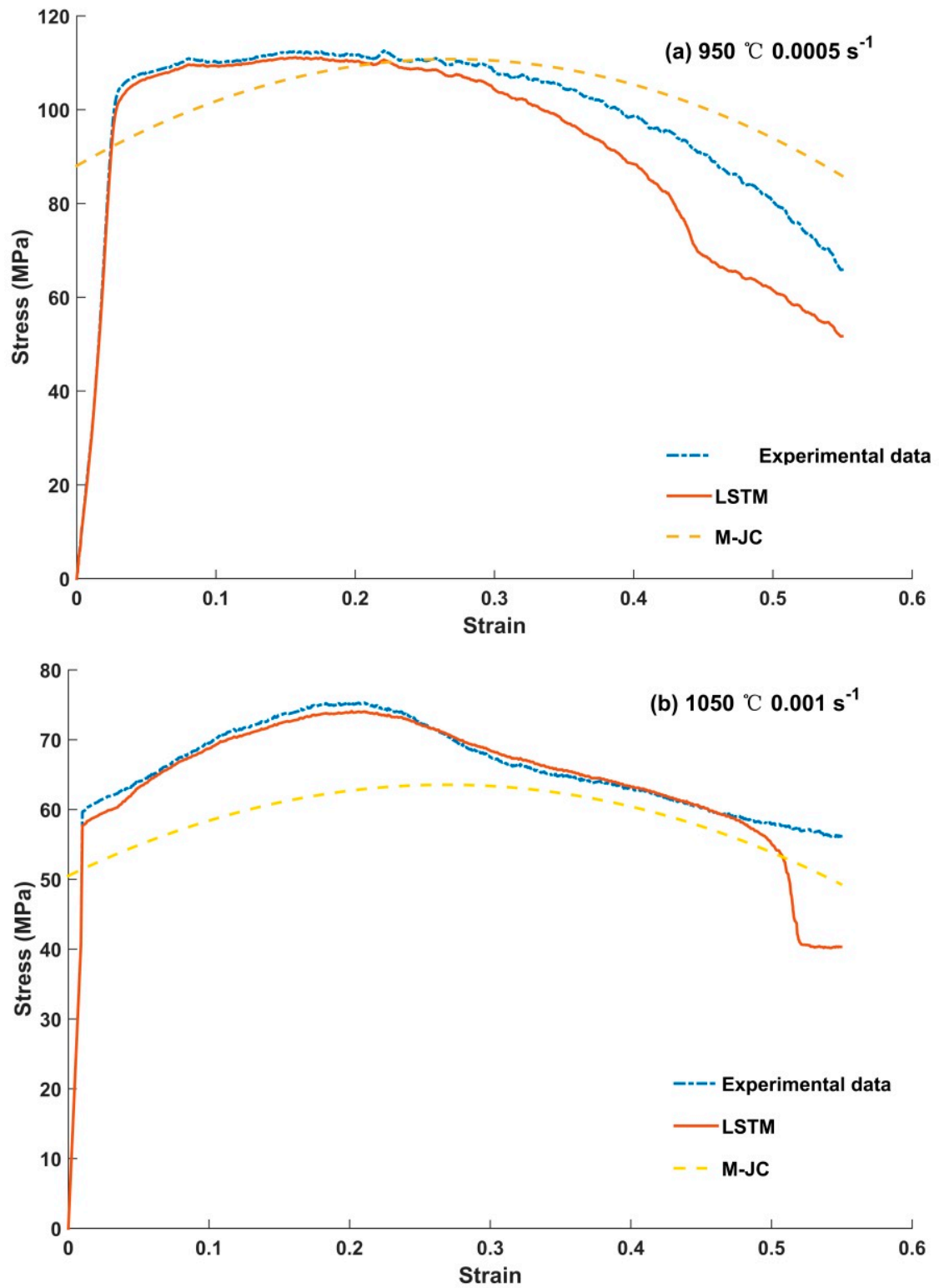

Figure 6. Prediction results of LSTM constitutive model and modified Johnson-Cook (M-JC) constitutive model. (a) $950{ }^{\circ} \mathrm{C}$; (b) $1050{ }^{\circ} \mathrm{C}$.
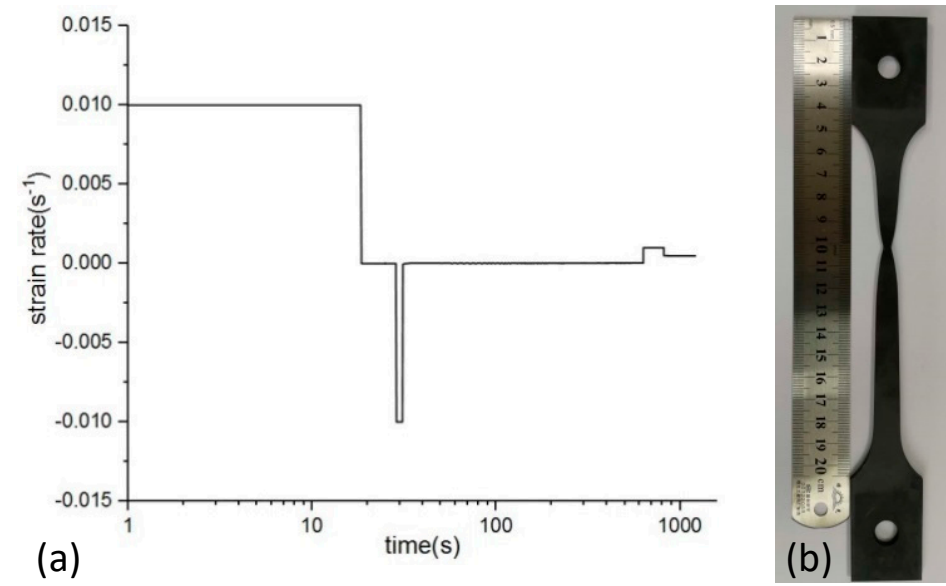

Figure 7. Complex loading tensile test of Inconel 718. (a) Strain rate loading curve; (b) Tensile specimen. 


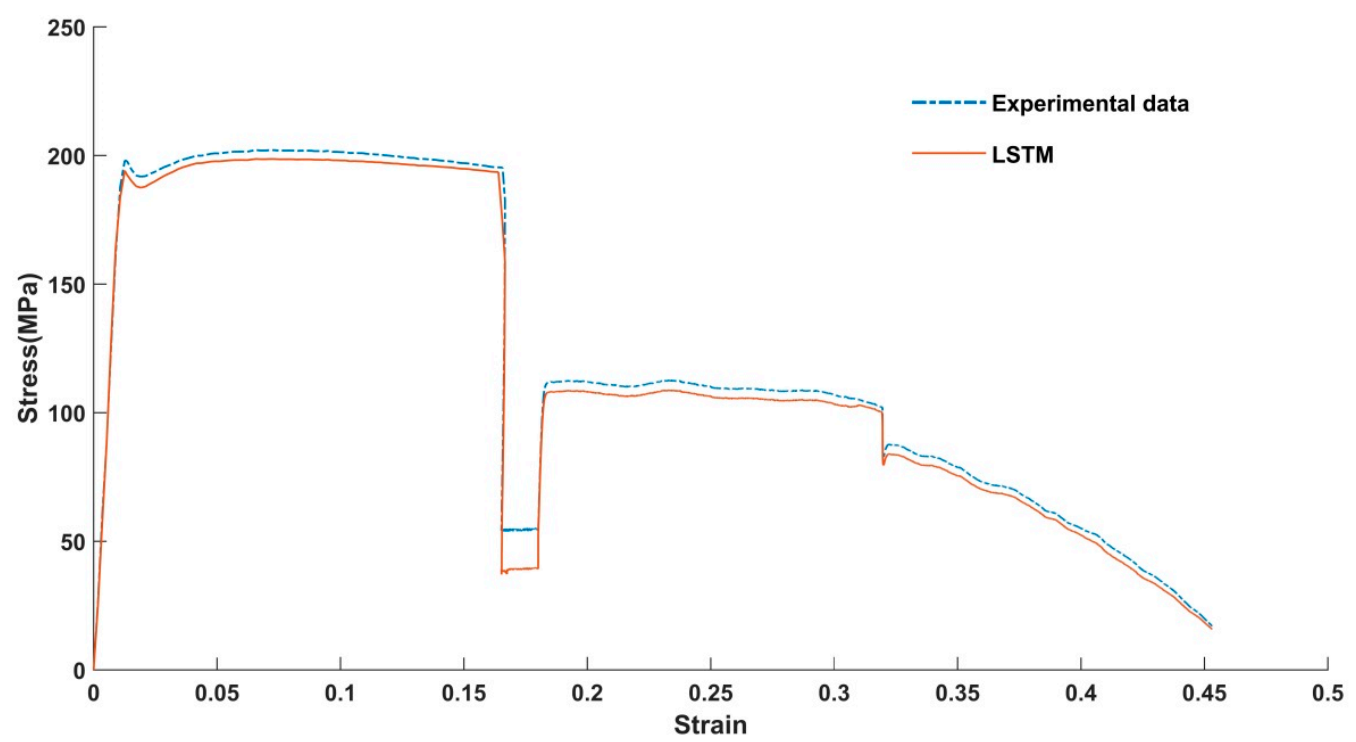

Figure 8. Comparison of predicted and measured results under complex loading paths.

\section{Conclusions}

The following conclusions can be derived from this study:

(1) Uniaxial tensile experiments were conducted on Inconel 718 at different temperatures and strain rates. Under different deformation conditions, the material exhibits obvious work hardening and cyclic softening coupling characteristics. Through the analysis of the true stress-true strain curve, it is found that the rheological behavior of Inconel 718 at high temperature has significant historical correlation with loading.

(2) A long short term memory recurrent neural network was trained using experimental data to characterize the constitutive relation considering the loading history effect. The model has a multi-hidden layer structure composed of LSTM layer and full connection layer. The Adam optimization method was adopted as the training algorithm, and the RMSE of training set after 603 iterations was 2.429 .

(3) The prediction accuracy of LSTM constitutive model was tested by using $950{ }^{\circ} \mathrm{C}, 0.0005 \mathrm{~s}^{-1}$ and $1050^{\circ} \mathrm{C}, 0.001 \mathrm{~s}^{-1}$ deformation parameters as the verification set. By comparing with the predicted results of M-JC model, it was found that the LSTM model can accurately predict the rheological behavior in both elastic and plastic stages. In the plasticity phase, the RMSE of predicted results by a LSTM model was only $32 \%$ and $20 \%$ of the M-JC model.

(4) Finally, the model is used to predict the deformation behavior under complex loads. The experimental results show that the LSTM constitutive model can accurately track the sudden change of the strain rate of Inconel 718 during high temperature deformation.

Author Contributions: H.M. and X.Y. conceived and designed the experiments; H.M. and Z.L. performed the experiments; H.M. and L.L. analyzed the data; H.M. wrote the paper; X.Y. and X.L. contributed to the revision of the paper. All authors have read and agreed to the published version of the manuscript.

Funding: This research received no external funding.

Conflicts of Interest: The authors declare no conflict of interest. 


\section{References}

1. Iyer, S.K.; Lissenden, C.J. Multiaxial constitutive model accounting for the strength-differential in inconel 718. Int. J. Plast. 2003, 19, 2055-2081. [CrossRef]

2. Lewandowski, J.J.; Wesseling, P.; Prabhu, N.S.; Larose, J.; Lerch, B.A. Strength differential measurements in IN718: Effects of superimposed pressure. Metall. Mater. Trans. A 2003, 34, 1736-1739. [CrossRef]

3. Sundararaman, M.; Mukhopadhyay, P.; Banerjee, S. Precipitation of the $\delta-\mathrm{Ni} 3 \mathrm{Nb}$ Phase in Two Nickel Base Superalloys. Metall. Mater. Trans. A 1988, 19, 453-465. [CrossRef]

4. Cozar, R.; Pineau, A. Morphology of $\gamma^{\prime}$ and $\gamma^{\prime \prime}$ precipitates and thermal stability of inconel 718 type alloys. Metall. Mater. Trans. B 1973, 4, 47-59. [CrossRef]

5. Thomas, A.; El-Wahabi, M.; Cabrera, J.M.; Prado, J.M. High temperature deformation of Inconel 718. J. Mater. Process. Technol. 2006, 177, 469-472. [CrossRef]

6. Fisk, M.; Ion, J.C.; Lindgren, L.E. Flow stress model for IN718 accounting for evolution of strengthening precipitates during thermal treatment. Comput. Mater. Sci. 2014, 82, 531-539. [CrossRef]

7. Wang, Y.; Shao, W.Z.; Zhen, L.; Yang, L.; Zhang, X.M. Flow behavior and microstructures of superalloy 718 during high temperature deformation. Mater. Sci. Eng. A 2008, 497, 479-486. [CrossRef]

8. Algarni, M.; Bai, Y.; Choi, Y. A study of Inconel 718 dependency on stress triaxiality and Lode angle in plastic deformation and ductile fracture. Eng. Fract. Mech. 2015, 147, 140-157. [CrossRef]

9. Iturbe, A.; Giraud, E.; Hormaetxe, E.; Garay, A.; Germain, G.; Ostolaza, K. Mechanical characterization and modelling of Inconel 718 material behavior for machining process assessment. Mater. Sci. Eng. A 2017, 682, 441-453. [CrossRef]

10. Lin, Y.C.; Li, K.K.; Li, H.B.; Chen, J.; Chen, X.M.; Wen, D.X. New constitutive model for high-temperature deformation behavior of inconel 718 superalloy. Mater. Des. 2015, 74, 108-118. [CrossRef]

11. Lin, Y.C.; Chen, X.M. A critical review of experimental results and constitutive descriptions for metals and alloys in hot working. Mater. Des. 2011, 32, 1733-1759. [CrossRef]

12. Lin, Y.C.; Wen, D.X.; Huang, Y.C.; Chen, X.M.; Chen, X.W. A unified physically based constitutive model for describing strain hardening effect and dynamic recovery behavior of a Ni-based superalloy. J. Mater. Res. 2015, 30, 3784-3794. [CrossRef]

13. Zhang, J.M.; Gao, Z.Y.; Zhuang, J.Y.; Zhong, Z.Y. Mathematical modeling of the hot-deformation behavior of superalloy IN718. Metall. Mater. Trans. A 1999, 30, 2701-2712. [CrossRef]

14. Kobayashi, T.; Simons, J.W.; Brown, C.S.; Shockey, D.A. Plastic flow behavior of Inconel 718 under dynamic shear loads. Int. J. Impact Eng. 2008, 35, 389-396. [CrossRef]

15. Lee, W.S.; Lin, C.F.; Chen, T.H.; Chen, H.W. Dynamic mechanical behaviour and dislocation substructure evolution of Inconel 718 over wide temperature range. Mater. Sci. Eng. A 2011, 528, 6279-6286. [CrossRef]

16. Wang, X.; Huang, C.; Zou, B.; Liu, H.; Zhu, H.; Wang, J. Dynamic behavior and a modified Johnson-Cook constitutive model of Inconel 718 at high strain rate and elevated temperature. Mater. Sci. Eng. A 2013, 580, 385-390. [CrossRef]

17. Hussain, M.Z.; Li, F.G.; Wang, J.; Yuan, Z.W.; Li, P.; Wu, T. Determination of Constitutive Equation for Thermo-mechanical Processing of INCONEL 718 through Double Multivariate Nonlinear Regression Analysis. J. Mater. Eng. Perform. 2015, 24, 2744-2756. [CrossRef]

18. Huang, Y.; Blackwell, P.L. Prediction of mechanical properties of superplastic Inconel* 718 using artificial neural networks. Mater. Sci. Technol. 2002, 18, 1104-1108. [CrossRef]

19. Yi, D.Q.; Wang, B.; Yao, C.G. Artificial neural network modelling to predict hot deformation behaviour of as HIPed FGH4169 superalloy. Mater. Sci. Technol. 2014, 30, 1170-1176.

20. Twala, O.A.B. Constitutive Modelling of INCONEL 718 using Artificial Neural Network. In IOP Conference Series Materials Science and Engineering; IOP Publishing: Bristol, UK, 2017; p. 225.

21. Pouranvari, M.; Ekrami, A.; Kokabi, A.H. Solidification and solid state phenomena during TLP bonding of IN718 superalloy using Ni-Si-B ternary filler alloy. J. Alloys Compd. 2013, 563, 143-149. [CrossRef]

22. Pouranvari, M.; Ekrami, A.; Kokabi, A.H. TLP bonding of cast IN718 nickel based superalloy: Process-microstructure-strength characteristics. Mater. Sci. Eng. A 2013, 568, 76-82. [CrossRef]

23. Gers, F.A.; Schmidhuber, J.; Cummins, F. Learning to Forget: Continual Prediction with LSTM. Neural Comput. 2000, 12, 2451-2471. [CrossRef] [PubMed] 
24. Bengio, Y. Learning long-term dependencies with gradient descent is difficult. IEEE Trans. Neural. Netw. 1994, 5, 157-166. [CrossRef] [PubMed]

25. Mei, H.; Lang, L.L.; Liu, K.N.; Yang, X.G. Evaluation Study on Iterative Inverse Modeling Procedure for Determining Post-Necking Hardening Behavior of Sheet Metal at Elevated Temperature. Metals 2018, 8, 1044. [CrossRef]

26. Lin, Y.C.; Chen, X.M.; Liu, G. A modified Johnson-Cook model for tensile behaviors of typical high-strength alloy steel. Mater. Sci. Eng. A 2010, 527, 6980-6986. [CrossRef]

27. Kotkunde, N.; Krishnamurthy, H.N.; Puranik, P.; Gupta, A.K.; Singh, S.K. Microstructure study and constitutive modeling of Ti-6Al-4V alloy at elevated temperatures. Mater. Des. 2014, 54, 96-103. [CrossRef]

Publisher's Note: MDPI stays neutral with regard to jurisdictional claims in published maps and institutional affiliations.

(C) 2020 by the authors. Licensee MDPI, Basel, Switzerland. This article is an open access article distributed under the terms and conditions of the Creative Commons Attribution (CC BY) license (http://creativecommons.org/licenses/by/4.0/). 